\title{
A Missense Mutation in Canine CLN6 in an Australian Shepherd with Neuronal Ceroid Lipofuscinosis
}

\author{
Martin L. Katz, ${ }^{1,2}$ Fabiana H. Farias, ${ }^{2}$ Douglas N. Sanders, ${ }^{1}$ Rong Zeng, ${ }^{2}$ Shahnawaz Khan, ${ }^{2}$ \\ Gary S. Johnson, ${ }^{2}$ and Dennis P. O'Brien ${ }^{3}$ \\ ${ }^{1}$ Mason Eye Institute, School of Medicine, College of Veterinary Medicine, University of Missouri, One Hospital Drive, \\ Columbia, MO 65212, USA \\ ${ }^{2}$ Department of Veterinary Pathobiology, College of Veterinary Medicine, University of Missouri, Columbia, MO 65211, USA \\ ${ }^{3}$ Department of Veterinary Medicine and Surgery, College of Veterinary Medicine, University of Missouri, \\ Columbia, MO 65211, USA
}

Correspondence should be addressed to Martin L. Katz, katzm@health.missouri.edu

Received 10 August 2010; Accepted 22 October 2010

Academic Editor: Monica Fedele

Copyright () 2011 Martin L. Katz et al. This is an open access article distributed under the Creative Commons Attribution License, which permits unrestricted use, distribution, and reproduction in any medium, provided the original work is properly cited.

The childhood neuronal ceroid lipofuscinoses (NCLs) are inherited neurodegenerative diseases that are progressive and ultimately fatal. An Australian Shepherd that exhibited a progressive neurological disorder with signs similar to human NCL was evaluated. The cerebral cortex, cerebellum, and retina were found to contain massive accumulations of autofluorescent inclusions characteristic of the NCLs. Nucleotide sequence analysis of DNA from the affected dog identified a T to C variant (c.829T $>C$ ) in exon 7 of CLN6. Mutations in the human ortholog underlie a late-infantile form of NCL in humans. The T-to-C transition results in a tryptophan to arginine amino acid change in the predicted protein sequence. Tryptophans occur at homologous positions in the CLN6 proteins from all 13 other vertebrates evaluated. The c.829T $>C$ transition is a strong candidate for the causative mutation in this NCL-affected dog. Dogs with this mutation could serve as a model for the analogous human disorder.

\section{Introduction}

The neuronal ceroid lipofuscinoses (NCLs) are inherited neurodegenerative disorders that occur in humans and a number of other mammals, including dogs, cats, sheep, mice, and cattle $[1,2]$. In most NCLs, the development of the nervous system appears to occur normally, but starting at various ages, progressive neurodegeneration leads to symptoms that can include vision loss, behavior changes, cognitive and motor decline, seizures, and premature death. The NCLs are distinguished from other inherited neurodegenerative disorders by the accumulation of autofluorescent lysosomal storage granules in neurons and other cell types, both within and outside the central nervous system.

NCLs have been reported in a large number of dog breeds including Border Collies, English Setters, American Bulldogs, Dachshunds, Polish Lowland Sheepdogs, and Tibetan Terriers [3]. For many breeds, NCLs have been reported as isolated cases and no genetic analyses have been undertaken. However, for five breeds (Border Collies, English Setters, American Bulldogs, Dachshunds, and Tibetan Terriers), the causative mutations have been identified, all of which are associated with an autosomal recessive mode of inheritance. These mutations all occur in orthologs of genes that contain mutations responsible for specific forms of human NCL or a similar disorder. We previously identified NCL in a family of Australian Shepherds [4]. Only paraffin-embedded tissues were available from these dogs, and no genetic analyses were undertaken. Formalin-fixed tissues were obtained from an unrelated Australian Shepherd that was euthanized after exhibiting similar symptoms. Ultrastructural examination of the storage materials in brain samples suggested that the disease in this dog might result from a CLN6 mutation. Therefore, genomic DNA was recovered from paraffin blocks from the dog's tissues and analyzed to determine whether CLN6 contained any potential disease-causing mutations. 
TABLE 1: PCR primer sequences used for analysis of canine the CLN6 gene.

\begin{tabular}{llc}
\hline Target & Forward primer/reverse primer & Amplicon size $(\mathrm{bp})$ \\
\hline Exon 1 & CCGTTCGTGCTTCCCGCAAC/GGACGCGCGGTGGATGGAC & 288 \\
Exon 2 & TCGAGTCCCCATACCTTTCTG/CACTGCTGGTAAGGGCTCTGTC & 245 \\
Exon 3 & TTCTGGGCCTGGGTGAACAC/CTGCTTCCACTGGCCTCCATGA & 243 \\
Exon 4 & GAGCCACGGCCTCCCTC/GACGGCCCGACCCACAA & 267 \\
Exon 5 & TGGAGGCGCCCTGCTG/TGCGCGCTGCCCTCTAGT & 281 \\
Exon 6 & CAGGACGGCTGGTGCAAG/CCAGGGCACGCACGTTT & 281 \\
Exon $7\left(5^{\prime}\right)$ & GTGGGTACAGCGAGGGATGCC/CCGGGTACTTCTTCCTGAGC & 285 \\
Exon $7\left(3^{\prime}\right)$ & CTTCGCTCTCACCCTCCTGCT/GCCGTGATGACCGCACA & 249 \\
\hline
\end{tabular}

\section{Materials and Methods}

A pet female Australian Shepherd began to exhibit decreased vision around the age of 18 months, and by 19 months this had progressed to complete blindness. At the same time the dog began to exhibit signs of anxiety, circling behavior, and loss of coordination that became progressively worse. The anxiety progressed as well, and she became very startled and anxious when touched or exposed to loud and sudden sounds. Ultimately her anxiety made her unwilling to go outside. She lost her ability to respond appropriately to her owners' voices. Eventually, it became difficult for her to locate her food or water dishes, and when she did, her lack of head coordination made it difficult for her to eat or drink. The dog was euthanized at 24 months of age, and the body was submitted to the Tennessee Department of Agriculture for pathological analysis. Gross examination of the brain revealed enlarged lateral ventricles and apparent hypoplasia of the cerebellum. Formalin-fixed samples of brain and retina were sent to us for analysis.

Pieces of the fixed tissues were washed in $0.17 \mathrm{M}$ sodium cacodylate, $\mathrm{pH}$ 7.4, embedded in Tissue Tek (Sakura, Torrance, CA), and frozen. Sections of the tissue were cut at a thickness of $5 \mu \mathrm{m}$ with a cryostat and mounted on glass slides. The sections were covered with $0.17 \mathrm{M}$ sodium cacodylate. Coverslips were placed over them and attached to the slides at the edges with adhesive. The sections were then examined and photographed with a Zeiss Axiophot microscope equipped for epifluorescence illumination [5]. Additional pieces of the tissues were washed in $0.17 \mathrm{M}$ sodium cacodylate, $\mathrm{pH}$ 7.4, and were then incubated with gentle agitation in a mixed aldehyde fixative [6] for a minimum of 24 hours. The samples were then processed for examination with transmission electron microscopy using a microwave-assisted technique [6]. Sections were examined with a JEOL 1400 transmission electron microscope.

Twenty sections were cut at a thickness of $20 \mu \mathrm{m}$ from a paraffin-embedded block of cerebral cortex from the NCL-affected Australian Shepherd. The sections were pooled, deparaffinized, and rehydrated. Genomic DNA was extracted from the rehydrated tissue as previously described $[5,7]$. The same procedure was used to extract DNA from a paraffin block containing tissue from an unrelated NCL-affected Australian Shepherd described previously in [4]. Additional canine DNA samples were obtained from the University of Missouri Canine DNA Repository (http://www.caninegeneticdiseases.net/). These samples were extracted from the blood of donor dogs that had not exhibited signs of NCL.

Oligo software (version 6.70) and sequences from build 2.1 of the canine genome reference sequence (http:// www.ncbi.nlm.nih.gov/projects/mapview/map_searchcgi? taxid=9615) were used to design PCR primers flanking the coding regions in each of the seven canine CLN6 exons (Table 1). Exons 2, 3, and 5 to 7 were amplified as previously described [7]; however, because of their high GC content, exon 1 and 4 were amplified with AccuPrime GC-Rich DNA Polymerase (Invitrogen). Purified PCR amplification products were sequenced with an Applied Biosystems 3730xl DNA analyzer. A TaqMan allelic discrimination assay was used to genotype the dogs at the putative disease locus described below. The PCR primer sequences were $5^{\prime}$-CCTCTTCTACTCCTTCGCTCTCA$3^{\prime}$ and $5^{\prime}$-ACACCCGGGTACTTCTTCCT- $3^{\prime}$ and the competing fluorescent labeled probe sequences were $5^{\prime}$ VIC-CAGGCGACCCGCAGG-MGB-3' (mutant) and $5^{\prime}$ FAM-CAGGCGACCCACAGG-MGB-3' (wild type).

\section{Results}

Fluorescence microscopic examination of unstained tissue sections revealed the presence of massive amounts of autofluorescent material in the cerebral cortex, cerebellum, and retina of the affected dog (Figure 1). This material was widely distributed throughout the cerebral cortex, but occurred most predominantly in the Purkinje and granular layers of the cerebellum. Autofluorescence in the retina occurred primarily in the ganglion cell layer (Figure 1). The ultrastructure of the storage bodies from each of the tissues appeared as membrane-bound inclusions filled with tightly packed vesicular and membranous structures (Figure 2).

Using the primers in Table 1, we were able to amplify all seven CLN6 exons with template DNA from blood from control dogs. Nonetheless, we were only able to amplify CLN6 exons 2, 3, 5, 6, and 7 (but not exons 1 and 4) from the DNA recovered from paraffin blocks containing tissue from the affected Australian Shepherd. A comparison of the sequences of the amplified exons from the affected dog with corresponding sequences from a clinically normal Australian Shepherd and with sequences in the canine 


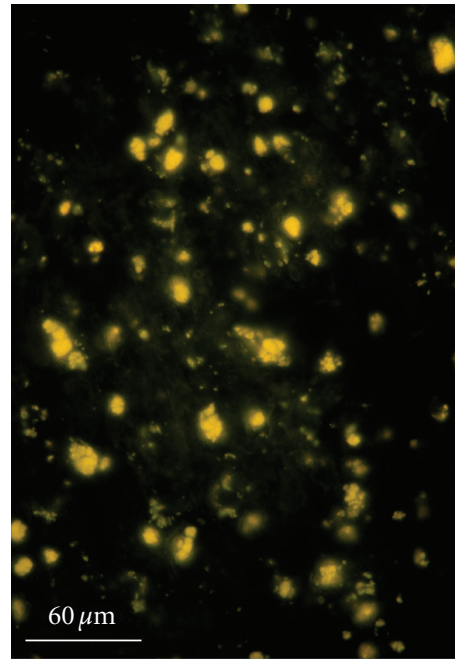

(a)

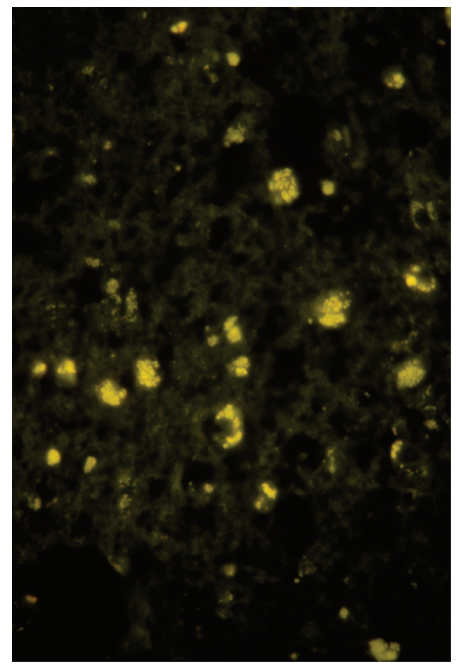

(b)

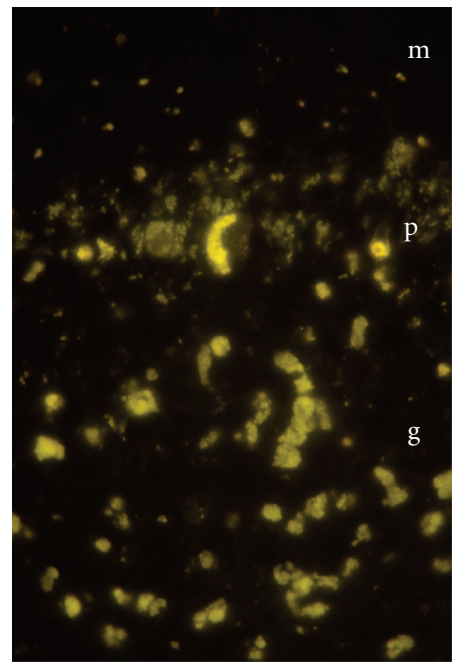

(c)

FIGURE 1: Fluorescence micrographs of the ganglion cell layer of the retina (a), cerebral cortex (b), and cerebellum (c) from the Australian Shepherd that was euthanized after exhibiting the neurological signs described in the text. In the cerebellum, autofluorescent material was most prominent in the Purkinje cell (p) and granular layers (g), with lesser accumulation in the molecular layer (m). Bar in (a) indicates magnification of all 3 micrographs.

genome reference sequence Build 2.1 (http://www.ncbi.nlm .nih.gov/projects/mapview/map_search.cgi?taxid=9615) revealed two sequence variants: a c.668+7G>A polymorphism in intron 6 and a c.829T $>C$ transition in exon 7 (Figure 3). The latter sequence change results in a missense mutation that predicts a p.W277R mutation in the canine CLN6 protein. Comparison of the predicted amino acid sequence of the affected dog with those of 13 other vertebrate species including mammals, birds, fish, and amphibians indicated that tryptophan was conserved at the equivalent site in all other vetebrates with available sequences (Figure 4(a)).

We used a TaqMan allelic discrimination assay, designed to distinguish wild-type homozygotes, heterozygotes, and mutant homozygotes, to genotype DNA samples from the NCL-affected Australian Shepherd, 637 healthy unrelated Australian Shepherds, and 43 dogs of other breeds from the University of Missouri Canine DNA Repository. The affected Australian Shepherd was homozygous for the $\mathrm{C}$ allele, but all of the other genotyped dogs were homozygous for the $\mathrm{T}$ allele. We were also able to genotype one of the Australian Shepherds previously reported to have suffered from NCL [4] using DNA recovered from paraffin sections. This affected Australian was also homozygous for the wild-type T allele.

\section{Discussion}

The clinical history and histopathologic findings confirm that the subject of this paper was affected with NCL. Of the NCLs, the ultrastructural appearance of the brain storage material most closely resembled that of storage bodies that accumulate in the brain as a result of mutations in CLN6 [8]. Therefore, this gene was analyzed for mutations that might underlie the NCL of the Australian Shepherd. While we cannot draw definitive conclusions about the genetic cause of

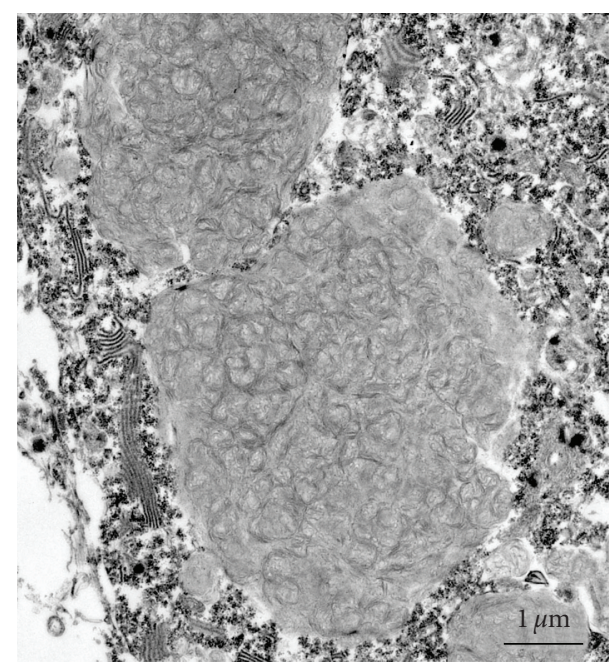

Figure 2: Electron micrograph of storage bodies from the cerebellum of the affected Australian Shepherd.

the disease based solely on DNA from a single dog, the $c .829 \mathrm{~T}>\mathrm{C}$ transition constitutes a missense mutation producing a CGG arginine codon instead of the common TGG codon for tryptophan. Because all vertebrate CLN6 sequences available in GenBank and CLN6 sequences of hundreds of unaffected dogs have a tryptophan at the corresponding position, this exon 7 mutation is a likely candidate for causing NCL in this Australian Shepherd. CLN6 is predicted to be an intrinsic membrane protein with 7 transmembrane domains [9]. Based on the predicted topology, the p.W277R mutation would lie within the membrane-spanning region of the 7 th transmembrane domain. Five missense mutations encoding a single amino 


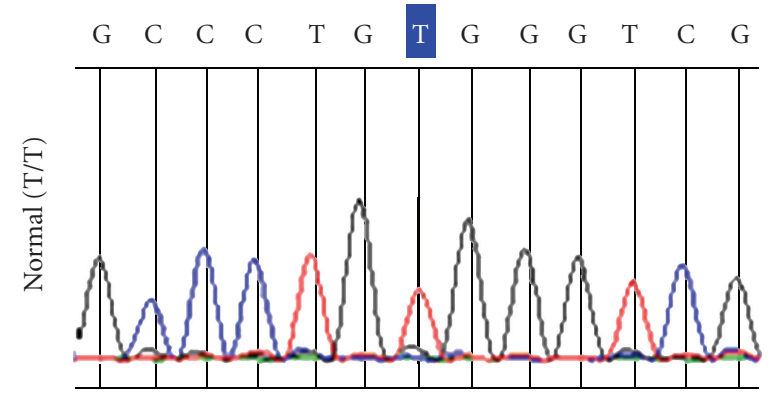

(a)

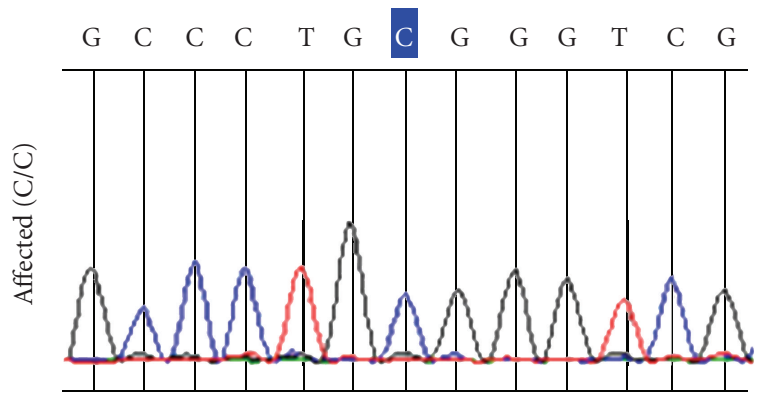

(b)

FIgUre 3: Partial nucleotide sequence of exon 7 of CLN6 in a normal dog and in the affected Australian Shepherd illustrating the c.829T $>C$ mutation.

\begin{tabular}{|c|c|}
\hline Homo sapiens & SNG L F L F S S F A L T L L L V A L $\underline{W} V$ AWLWND P V L R K K Y P GV I Y V P E PWA F \\
\hline Pan troglodytes & S NG L F L F S S F T L T L L L V A L $\underline{W V A W L W N D ~ P ~ V ~ L ~ R ~ K ~ K ~ Y ~ P ~ G V ~ I ~ Y ~ V ~ P ~ E ~ P W A ~ F ~}$ \\
\hline Macaca mulatta & S NG L F L F S S F T L T L L L V A L WV AWLWND P V L R K K Y P GV I Y V P E PWA F \\
\hline Bos taurus & SNG L F L F Y S F A L T L L L V A L $\underline{W V A W L W N D P V ~ L ~ R ~ K K ~ Y ~ P ~ G V ~ I ~ Y V ~ P ~ E ~ P W A ~ F ~}$ \\
\hline Rattus norvegicus & SNG L F L F C S F A L T L L L V A L $\underline{W V A W L W N D P V L R K K Y ~ P ~ G V ~ I ~ Y V ~ P ~ E ~ P W A ~ F ~}$ \\
\hline Mus musculus & SNG L F L L Y S F A L T L S L V A L $\underline{W V A W L W N D P V ~ L ~ R K K Y ~ P ~ G V ~ I ~ Y V ~ P ~ E ~ P W A ~ F ~}$ \\
\hline Ovis aries & SNG L F L F Y S F A L A L L L V A L WVVAWLWND PV L R KKY P GV I YV P E PWA F \\
\hline Ornithorhynchus anatinus & S NGL F L F Y S F V L T L V L V A L $\underline{W V G W L W N D P ~ T ~ L ~ R ~ K ~ K Y ~ P ~ G V ~ I ~ Y V P ~ E ~ P W A ~ F ~}$ \\
\hline Gallus gallus & SNGL F L F Y S F I I T L V L I A VWVVVWLWNDKT LRKKY P GV I Y I P E PWA F \\
\hline Xenopus laevis & SNGL F L L Y S F S A T L I F I A AW I VWLWNDQ I L R KKY P GV I Y I P E PWA F \\
\hline Xenopus tropicalis & 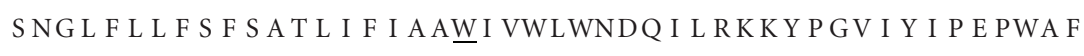 \\
\hline Danio rerio & SNG L F L L Y S F S I S L F L V S VWWV A C LWNDKV L R KKY P GVMY I P E PWA L \\
\hline Tetraodon nigroviridis & ANG L F V L S S F L A A L L L V A V $\underline{W V ~ L W L W D D G V ~ L ~ R ~ K K H P G ~ L ~ I ~ Y ~ V ~ P ~ Q ~ P ~ R ~ T ~ V ~}$ \\
\hline Canis lupus familiaris & SNG L F L F Y S F A L T L L L V A L $\underline{W V A W L W N D P V ~ L ~ R ~ K K Y ~ P ~ G V ~ I ~ Y V ~ P ~ E ~ P W A ~ F ~}$ \\
\hline Canis lupus & NGL F L F Y S F A L T L L L V A L R VAWLWND P V L R K K Y P GV I Y V P E PWA F \\
\hline
\end{tabular}

(a)

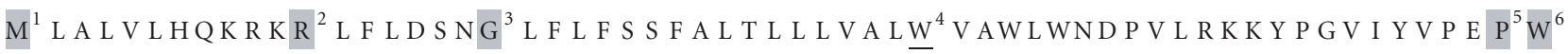

Figure 4: (a) Partial amino acid sequences of CLN6 from a number of species showing uniqueness of the p.W277R mutation in the affected Australian Shepherd. (b) Partial amino acid sequence of human CLN6 showing missense mutation sites (shaded: p.M241T ${ }^{1}$; p.R252H ${ }^{2}$; pG259C 3 ; p.P299L ${ }^{5}$; p.W300R ${ }^{6}$ ) that result in human vLINCL and the amino acid corresponding to the Australian Shepherd mutation (underlined: p.W277R $\mathrm{R}^{4}$ ).

acid change have been documented in human patients for this region of CLN6 (Figure 4(b)), all of which cause the same variant form of late infantile NCL (vLINCL) (http://www.ucl.ac.uk/ncl/cln6.shtml). Because the function of CLN6 is currently unknown [10], it is not possible to predict what effects this mutation might have on cellular metabolism or to determine whether the mutation results in functional alterations.

Our inability to amplify and sequence exons 1 and 4 of CLN6 from the affected Australian Shepherd could be attributable to partial degradation of the DNA recovered from formalin-fixed tissue. However, the possibility that these exons contain additional sequence variants cannot be ruled out. The c. $668+7 \mathrm{G}>\mathrm{A}$ polymorphism in Intron 6 is unlikely to be the cause of the disease as it is beyond the consensus sequence for exon splice donor recognition [11].

Unfortunately, pedigree information from the affected Australian shepherd was unavailable and closely related dogs could not be located, so we were unable to genotype other 
dogs from the same family. We failed to identify additional dogs carrying the mutant allele even though we screened 680 dogs including 637 Australian Shepherds. The only other published report of NCL in Australian Shepherds described NCL in three littermates with ages at onset and general patterns of symptoms that were very similar to that of the Australian Shepherd described above [4]. We extracted DNA from a paraffin block containing brain tissue from one of the affected littermates and determined that the dog was homozygous for the wild-type $\mathrm{T}$ allele. This suggests that two distinct forms of NCL have occurred among Australian Shepherds. Consistent with this possibility, the pattern of autofluorescent storage material in the cerebellum was quite different between the current dog and the affected Australian Shepherd littermates that were previously described. In the currently described Australian Shepherd, the vast majority of the storage material was present in the granular cell layer, whereas in the previous cases the storage material occurred primarily in the Purkinje cells (cf. Figures 2 and 5) [4]. In addition, there were distinct differences in the ultrastructural appearance of the storage bodies between the dog with CLN6 mutation and those of the Australian Shepherds previously described with NCL [4]. Whereas in the present case the storage bodies appeared to be aggregates of membranous vesicles (Figure 2), the brain storage bodies in the previous cases consisted primarily of aggregates of multilayered membranous whorls [4]. In the Australian Shepherd evaluated in this study the ultrastructural appearance of the storage material was quite similar to that of storage material from the brains of sheep that have been shown to suffer from NCL as a result of a mutation affecting CLN6 expression $[8,12,13]$.

The occurrence of more than one form of NCL within a single breed is not surprising. We have identified two mutations responsible for NCL in Dachshunds [7, 14], as well as additional Dachshunds with NCL that have neither of these mutations. Likewise, similar disease phenotypes occur in humans with mutations in different genes that lead to NCL $[15,16]$. For a number of the human NCLs, it was not until the causative mutations were identified that it was recognized that many cases of NCL with similar symptoms and ages of onset actually represent different genetic defects. With the discovery of at least eight different NCL genes, several human NCLs that were previously classified together have now been shown to be genetically distinct. Therefore, even within a dog breed, the failure to detect a known NCL mutation is not sufficient to rule out NCL as the cause of progressive neurological deterioration.

Among Australian Shepherds, the frequency of the c. $829 \mathrm{~T}>\mathrm{C}$ allele appears to be very low, based on our survey of unaffected representatives of the breed. Further, the paucity of published reports suggests that NCL is rare in the breed. Thus, widespread testing for the CLN6 mutation is not presently warranted with respect to eliminating a potential health problem in the breed. However, given that symptom onset of both forms of NCL in this breed is less than 2 years, it would be wise for breeders to monitor any dogs they produce for signs of the disease for their first 2 years. If potentially

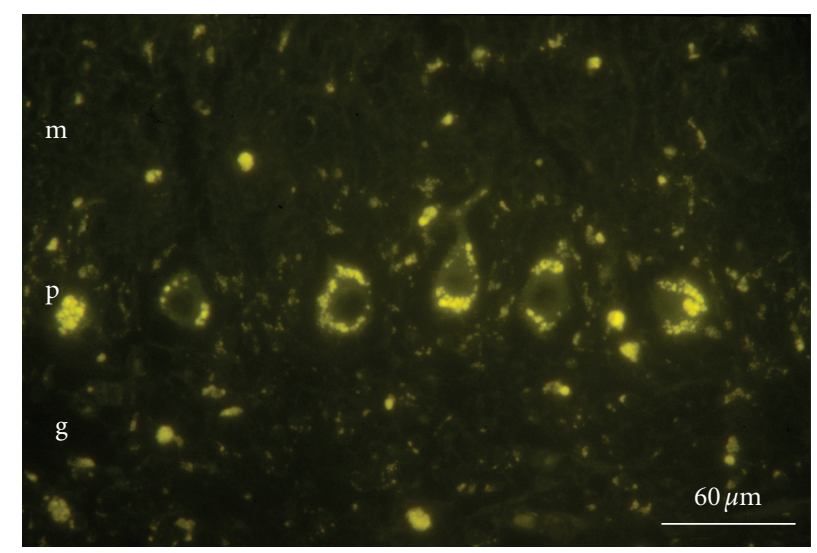

FIGURE 5: Fluorescence micrograph of the cerebellum from an Australian Shepherd with NCL that tested homozygous for the T allele at the c.829 locus [4]. The layers of the cerebellum are labeled as follows: g: granular layer; p: Purkinje layer; m: molecular layer.

affected dogs are identified, the affected dogs as well as the parents and other dogs closely related to the affected animals should be tested for the mutant allele. If living sexually intact dogs that are heterozygous for the CLN6 mutation can be located, they could be used for breeding to establish a research colony. Locating such dogs could be accomplished by screening large numbers of Australian Shepherds for the mutation using the allelic discrimination assay.

\section{Conclusions}

Australian Shepherds with the identified CLN6 mutation would be a valuable model for elucidating the function of the CLN6 protein and the mechanisms by which mutations in CLN6 result in NCL disease pathology. Such dogs would be useful for evaluating potential therapies for the corresponding human disease. Using an allelic discrimination assay, we should be able to identify those rare Australian Shepherds that carry the mutation for use in establishing a canine model.

We recently identified a living Australian shaped exhibiting clinical signs similar to those displayed by the dog described in this study. The living dog is homozygous for the C allele at c.829.

\section{Acknowledgments}

This work was supported by American Kennel Club Canine Health Foundation Grants 732 and 762, by the Batten Disease Support and Research Association, by an unrestricted grant from Research to Prevent Blindness, Inc., and by the University of Missouri PRIME Fund. The authors thank Liz Hansen for maintaining the DNA Repository from which the normal Australian Shepherd samples were obtained, the staff of the University of Missouri Electron Microscopy Core facility for their assistance, and the dogs' owners for providing us with the material necessary for this study. 


\section{References}

[1] R. D. Jolly and D. N. Palmer, "The neuronal ceroidlipofuscinoses (Batten disease): comparative aspects," Neuropathology and Applied Neurobiology, vol. 21, no. 1, pp. 50-60, 1995.

[2] M. L. Katz, H. Shibuya, and G. S. Johnson, "Animal models for the ceroid lipofuscinoses," Advances in Genetics, vol. 45, pp. 183-203, 2001.

[3] M. L. Katz, G. S. Johnson, and C. Drögemüller, "Canine neuronal ceroid lipofuscinoses," in The Neuronal Ceroid Lipofuscinoses (Batten Disease), S. E. Mole, B. D. Lake, and H. H. Goebel, Eds., IOS Press, London, UK, In Press.

[4] D. P. O'Brien and M. L. Katz, "Neuronal ceroid lipofuscinosis in 3 australian shepherd littermates," Journal of Veterinary Internal Medicine, vol. 22, no. 2, pp. 472-475, 2008.

[5] M. L. Katz, S. Khan, T. Awano, S. A. Shahid, A. N. Siakotos, and G. S. Johnson, "A mutation in the CLN8 gene in English Setter dogs with neuronal ceroid-lipofuscinosis," Biochemical and Biophysical Research Communications, vol. 327, no. 2, pp. 541547, 2005.

[6] K. D. Wendt, C. A. Jensen, R. Tindall, and M. L. Katz, "Comparison of conventional and microwave-assisted processing of mouse retinas for transmission electron microscopy," Journal of Microscopy, vol. 214, no. 1, pp. 80-88, 2004.

[7] T. Awano, M. L. Katz, D. P. O’Brien et al., "A frame shift mutation in canine TPP1 (the ortholog of human CLN2) in a juvenile Dachshund with neuronal ceroid lipofuscinosis," Molecular Genetics and Metabolism, vol. 89, no. 3, pp. 254-260, 2006.

[8] N. A. Hall, R. D. Jolly, D. N. Palmer, B. D. Lake, and A. D. Patrick, "Analysis of dolichyl pyrophosphoryl oligosaccharides in purified storage cytosomes from ovine ceroidlipofuscinosis," Biochimica et Biophysica Acta, vol. 993, no. 2-3, pp. 245-251, 1989.

[9] R. B. Wheeler, J. D. Sharp, R. A. Schultz, J. M. Joslin, R. E. Williams, and S. E. Mole, "The gene mutated in variant late-infantile neuronal ceroid lipofuscinosis (CLN6) and in nclf mutant mice encodes a novel predicted transmembrane protein," American Journal of Human Genetics, vol. 70, no. 2, pp. 537-542, 2002.

[10] A. Kurze, G. Galliciotti, C. Heine, S. E. Mole, A. Quitsch, and T. Braulke, "Pathogenic mutations cause rapid degradation of lysosomal storage disease-related membrane Protein CLN6," Human Mutation, vol. 31, no. 2, pp. 1163-1174, 2010.

[11] M. B. Shapiro and P. Senapathy, "RNA splice junctions of different classes of eukaryotes: sequence statistics and functional implications in gene expression," Nucleic Acids Research, vol. 15, no. 17, pp. 7155-7174, 1987.

[12] M. F. Broom, C. Zhou, J. E. Broom, K. J. Barwell, R. D. Jolly, and D. F. Hill, "Ovine neuronal ceroid lipofuscinosis: a large animal model syntenic with the human neuronal ceroid lipofuscinosis variant CLN6," Journal of Medical Genetics, vol. 35, no. 9, pp. 717-721, 1998.

[13] I. Tammen, P. J. Houweling, T. Frugier et al., "A missense mutation (c. 184C > T) in ovine CLN6 causes neuronal ceroid lipofuscinosis in Merino sheep whereas affected South Hampshire sheep have reduced levels of CLN6 mRNA," Biochimica et Biophysica Acta, vol. 1762, no. 10, pp. 898-905, 2006.

[14] D. N. Sanders, F. H. Farias, G. S. Johnson et al., "A mutation in canine PPT1 causes early onset neuronal ceroid lipofuscinosis in a Dachshund," Molecular Genetics and Metabolism, vol. 100, no. 4, pp. 349-356, 2010.
[15] E. Siintola, A. E. Lehesjoki, and S. E. Mole, "Molecular genetics of the NCLs—status and perspectives," Biochimica et Biophysica Acta, vol. 1762, no. 10, pp. 857-864, 2006.

[16] K. E. Wisniewski, E. Kida, A. A. Golabek, W. Kaczmarski, F. Connell, and N. Zhong, "Neuronal ceroid lipofuscinoses: classification and diagnosis," Advances in Genetics, vol. 45, pp. 1-34, 2001. 


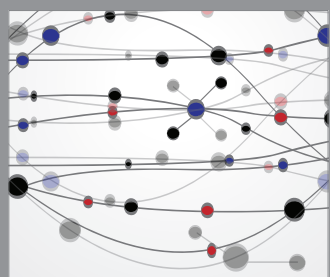

The Scientific World Journal
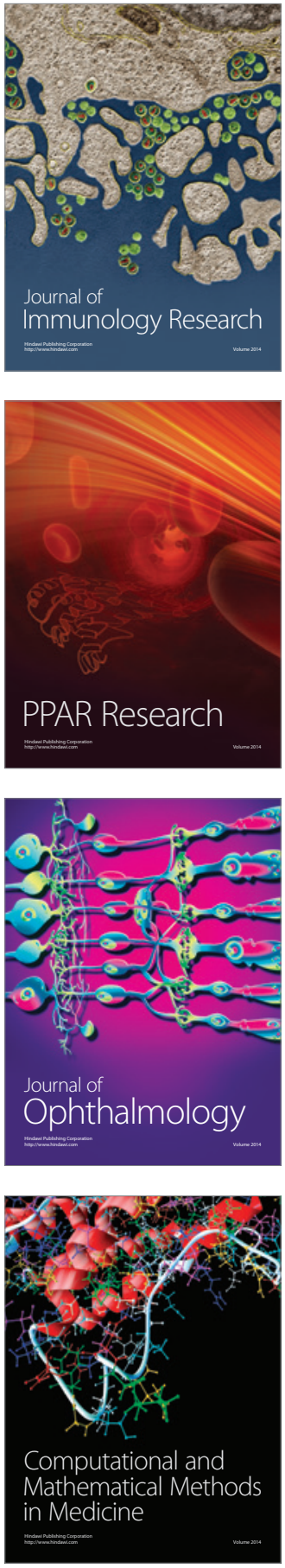

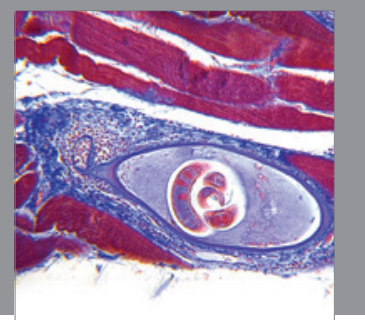

Gastroenterology

Research and Practice
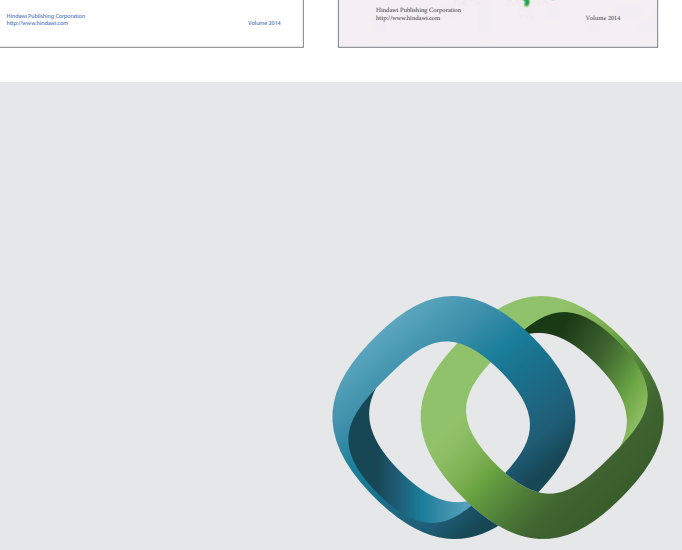

\section{Hindawi}

Submit your manuscripts at

http://www.hindawi.com
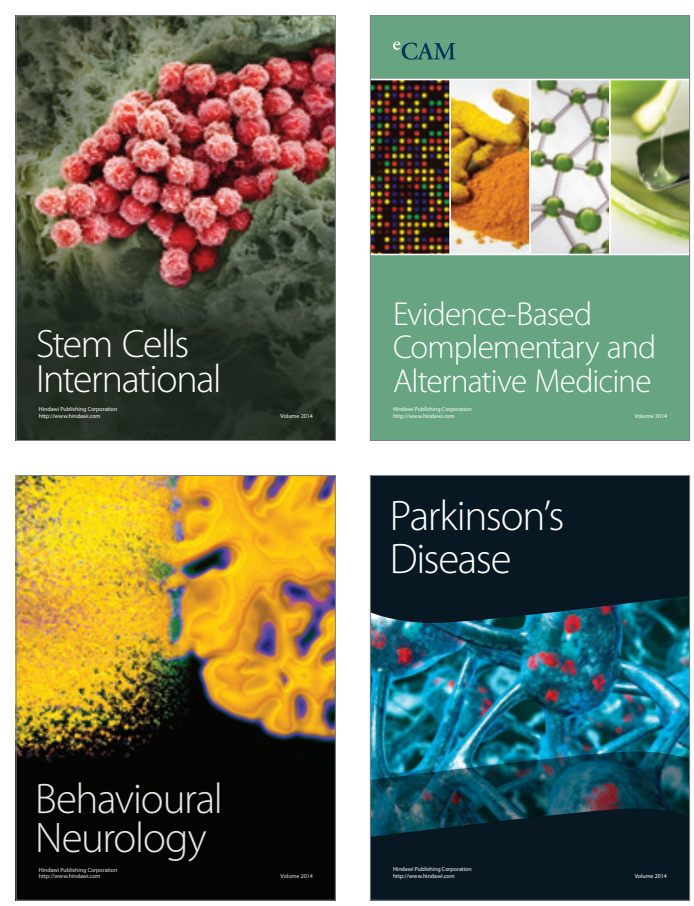

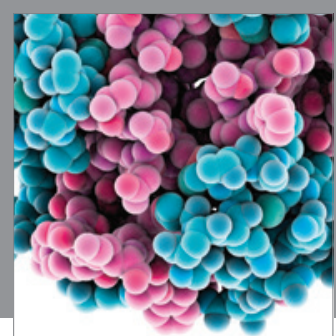

Journal of
Diabetes Research

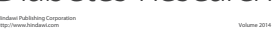

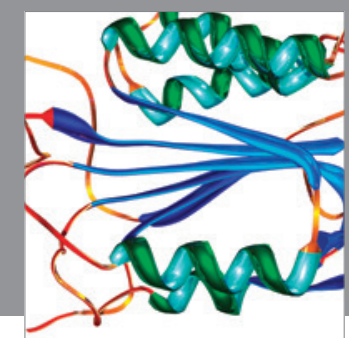

Disease Markers
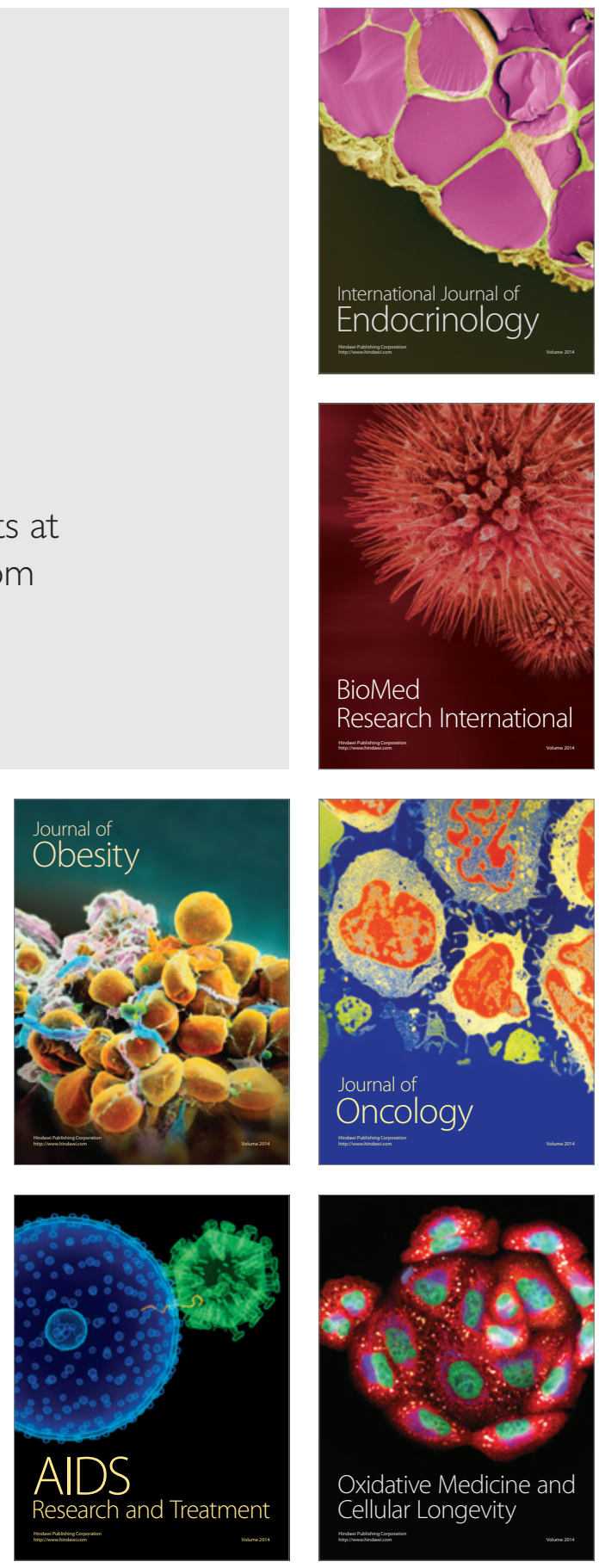